\title{
Expanding the phenotype of PRPS1 syndromes in females: neuropathy, hearing loss and retinopathy
}

Berta Almoguera ${ }^{1 \dagger}$, Sijie He ${ }^{2,3+}$, Marta Corton ${ }^{4,5}$, Patricia Fernandez-San Jose ${ }^{4,5}$, Fiona Blanco-Kelly ${ }^{4,5}$, Maria Isabel López-Molina, ${ }^{5,6}$, Blanca García-Sandoval ${ }^{5,6}$, Javier del Val ${ }^{7}$, Yiran Guo ${ }^{1}$, Lifeng Tian ${ }^{1}$, Xuanzhu Liư ${ }^{3}$, Liping Guan ${ }^{3}$, Rosa J Torres ${ }^{8}$, Juan G Puig ${ }^{9}$, Hakon Hakonarson ${ }^{1}$, Xun X Xu', ${ }^{3,10}$, Brendan Keating ${ }^{1+}$

and Carmen Ayuso ${ }^{4,5^{*}+}$

\begin{abstract}
Background: Phosphoribosyl pyrophosphate synthetase (PRS) I deficiency is a rare medical condition caused by missense mutations in PRPS1 that lead to three different phenotypes: Arts Syndrome (MIM 301835), X-linked Charcot-Marie-Tooth (CMTX5, MIM 311070) or X-linked non-syndromic sensorineural deafness (DFN2, MIM 304500). All three are X-linked recessively inherited and males affected display variable degree of central and peripheral neuropathy. We applied whole exome sequencing to a three-generation family with optic atrophy followed by retinitis pigmentosa (RP) in all three cases, and ataxia, progressive peripheral neuropathy and hearing loss with variable presentation.
\end{abstract}

Methods: Whole exome sequencing was performed in two affecteds and one unaffected member of the family. Sanger sequencing was used to validate and segregate the 12 candidate mutations in the family and to confirm the absence of the novel variant in PRPS1 in 191 controls. The pathogenic role of the novel mutation in PRPS1 was assessed in silico and confirmed by enzymatic determination of PRS activity, mRNA expression and sequencing, and X-chromosome inactivation.

Results: A novel missense mutation was identified in PRPS1 in the affected females. Age of onset, presentation and severity of the phenotype are highly variable in the family: both the proband and her mother have neurological and ophthalmological symptoms, whereas the phenotype of the affected sister is milder and currently confined to the eye. Moreover, only the proband displayed a complete lack of expression of the wild type allele in leukocytes that seems to correlate with the degree of PRS deficiency and the severity of the phenotype. Interestingly, optic atrophy and RP are the only common manifestations to all three females and the only phenotype correlating with the degree of enzyme deficiency.

Conclusions: These results are in line with recent evidence of the existence of intermediate phenotypes in PRS-I deficiency syndromes and demonstrate that females can exhibit a disease phenotype as severe and complex as their male counterparts.

Keywords: PRPS1, Retinitis pigmentosa, Non-random X-chromosome inactivation, Phosphoribosyl pyrophosphate synthetase deficiency, Neuropathy

\footnotetext{
* Correspondence: cayuso@fjd.es

${ }^{\dagger}$ Equal contributors

${ }^{4}$ Department of Genetics and Genomics, IIS-Fundación Jiménez Díaz

University Hospital (IISFJD, UAM), 28040 Madrid, Spain

${ }^{5}$ Center for Biomedical Network Research on Rare Diseases (CIBERER), ISCIII,

Madrid, Spain

Full list of author information is available at the end of the article
} 


\section{Background}

Phosphoribosyl pyrophosphate synthetase 1 (PRS-I, [MIM 311850]) is an ubiquitous enzyme with an essential role in nucleotide metabolism: it catalyzes the synthesis of phosphoribosyl pyrophosphate (PRPP), the substrate for the synthesis of purine, pyridine, and pyrimidine nucleotides [1]. PRS-I has two highly homologous isoforms, PRS-II (MIM 311860) widely expressed as PRS-I, and PRS-III (MIM 611566) whose expression is restricted to testes. The three isoforms are products of separate but highly conserved genes: PRPS1 (Xq22.3), PRPS2 (Xp22.2), and PRPS1L1 (7p21.1), respectively [2]. Missense mutations in PRPS1 are rare and may result in increase or decrease in PRS-I activity. PRS-I deficiency is an extremely rare condition that can lead to three different disorders: Arts syndrome (MIM 301835), being the most extreme form of enzyme deficiency; Rosenberg-Chutorian syndrome or X-linked Charcot-Marie Tooth 5 (CMTX5, MIM 311070), which represents a less severe phenotype; and X-linked non-syndromic sensorineural deafness (DFN2, MIM 304500), as the mildest form of deficiency. Only twelve families have been described so far with PRS-I deficiency syndromes [3-7] [I. del Castillo, personal communication]. Hearing loss is the only common feature between the three disorders and the only symptom observed in DFN2. Arts syndrome and CMTX5 share additional neurological anomalies such as ataxia, hypotonia, and optic atrophy $[4,8,9]$. Arts syndrome is also characterized by delayed motor development, mild to moderate intellectual disability and frequent recurrent infections that typically result in early death $[3,10]$. PRS-I superactivity (MIM 300661) is more frequent than the deficiency and is characterized by hyperuricemia and gout and it can be accompanied by other neurological symptoms such as sensorineural deafness, hypotonia, mental retardation, and also by recurrent infections [11,12]. All four disorders are inherited in a recessive X-linked manner so only males are affected. Obligate female carriers, however, may occasionally display milder symptoms such as hearing loss in CMTX5 $[8,9]$, hearing impairment, ataxia, hypotonia or hyperreflexia in Arts syndrome [3], or hyperuricemia in PRS-I superactivity [13].

Using whole exome sequencing (WES), we identified a novel loss-of-function mutation in PRPS1 leading to enzyme deficiency in three females with optic atrophy (OA), retinitis pigmentosa (RP), ataxia, peripheral neuropathy and hearing loss with variable presentation.

\section{Methods}

\section{Subjects}

A three-generation Spanish family (RP-0482) consisting of four affected females (Figure 1A) was recruited and evaluated by the Fundacion Jimenez Diaz Hospital (Madrid, Spain). The four affecteds, six unaffecteds and two unrelated members of the family participated in the genetic study. All four affected females, II:2, III:2, IV:2 and IV:3, displayed typical or sectorial RP and various degrees of neurological symptoms. II: 2 and II:3 died during the course of the study but we obtained their DNA samples and informed consent before allowing us to include them in the study. Additionally, 191 unrelated Spanish individuals with no history of retinal dystrophy and randomly selected from blood donors voluntarily participated as controls. Informed consent was obtained from all individuals involved, all procedures were reviewed and approved by the Ethics Committee of the Hospital and adhered to the tenets of the Declaration of Helsinki.

\section{Whole exome sequencing and variant analysis}

Genomic DNA was captured by Agilent SureSelect Human All Exon kit version 2 covering $46 \mathrm{MB}$ of coding region (Agilent Technologies, Santa Clara, CA, USA), and sequenced on HiSeq 2000 instruments (Illumina, San Diego, CA, USA). Raw reads were mapped to the human reference genome (UCSC hg19), using the Burrows-Wheeler alignment tool [15]. Genome Analysis Tool Kit version 1.4 [16] was used for variant calling. ANNOVAR [17] was used for variant functional annotation and prediction and conservation scores from SIFT [18], Polyphen2 [19], LRT [20], MutationTaster [21], PhyloP [22] and GERP++ [23] were retrieved from the Database for Nonsynonymous SNPs' Functional Predictions [24] for every potential nonsynonymous single nucleotide variant (SNVs).

Variant filtering was performed under the assumption of dominant inheritance. Only nonsynonymous, splice site disrupting, and frameshift heterozygous variants segregating in the family with a minor allele frequency $\leq 0.5 \%$ in a control cohort of more than 8000 individuals (1000 Genomes Project, [25] (April, 2012 release); 6503 exomes from NHLBI GO Exome Sequencing Project [26], and 669 in-house whole-exomes) were considered. Further gene prioritization was performed combining data on minor allele frequency, sequence conservation, potential deleterious effect and biological and clinical relevance according to gene function, gene expression, and the existence of mutation reports in databases such as The Human Gene Mutation Database [27], The Retinal Information Network (RetNet) [28] or Online Mendelian Inheritance in Man (OMIM) [29].

\section{Sanger sequencing}

Sanger sequencing was used for the segregation of all variants resulting from the filtering analysis in family RP-0482 and to confirm the absence of variant p.Ser16Pro in PRPS1 in 191 Spanish controls (primers available in Additional file 1). PCR products were enzymatically purified with ExoSAP-it (USB, Affymetrix), sequenced on both strands using Big Dye Terminator Cycle Sequencing 


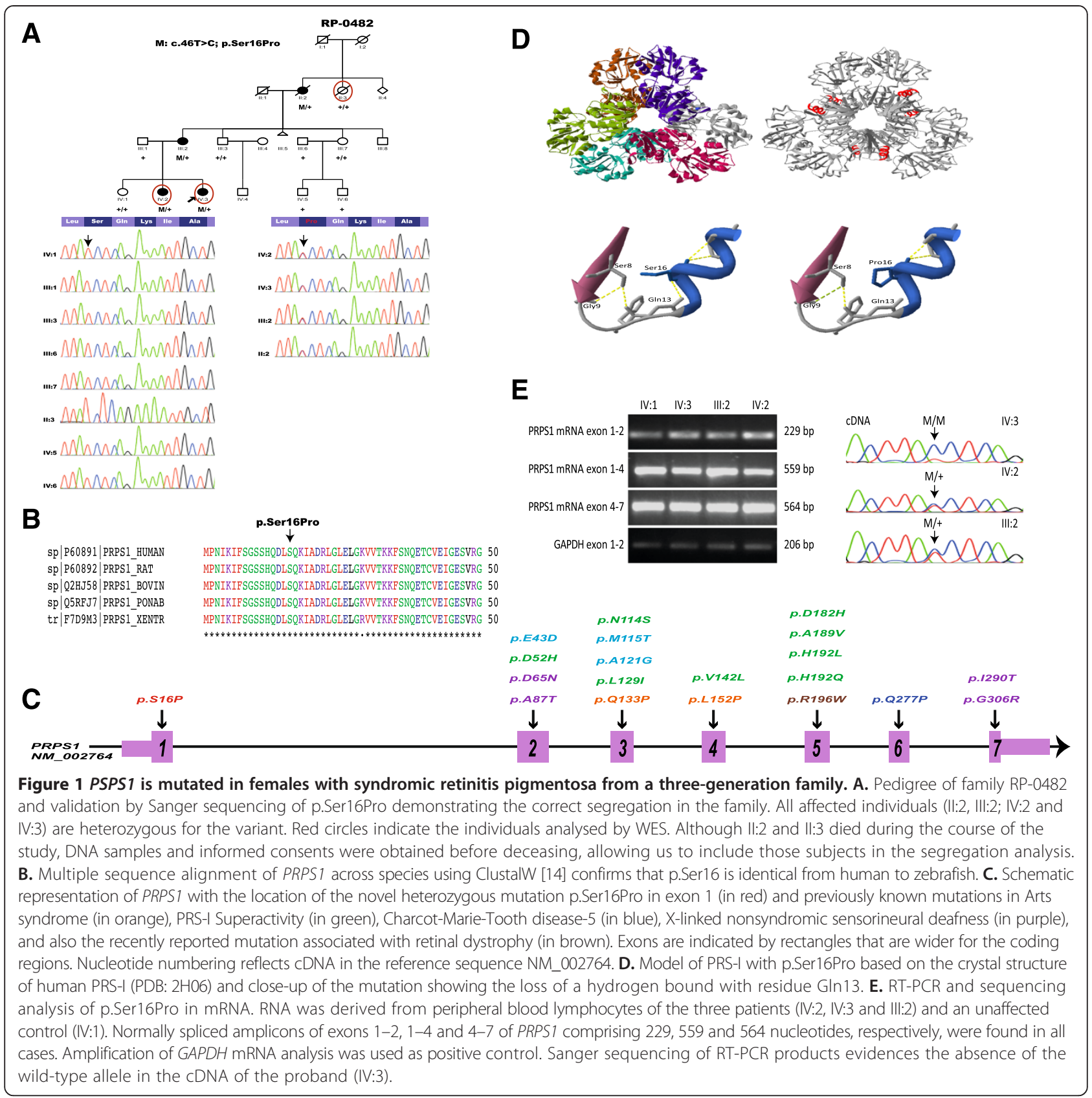

Kit v3.1 (Applied Biosystems) and resolved on an automated sequencer (ABI 3130xl Genetic Analyzer, Applied Biosystems).

\section{In silico analyses of p.Ser16Pro pathogenicity}

The evolutionary conservation of p.Ser16Pro was assessed by multiple sequence alignment of PRPS1 across species using ClustalW2 [14]. The impact of the mutation in the tridimensional structure of the protein was assessed with a model of PRS-I with p.Ser16Pro based on the crystal structure of the human protein (PDB: 2H06) using Swiss Model [30,31] and Swiss PDB viewer [32]. ESEFinder
$[33,34]$ was used to determine whether p.Ser16Pro could alter the normal splicing of the mRNA.

\section{Determination of PRS activity in erythrocytes}

PRS enzymatic activity was determined in erythrocytes from III:2, III:3, IV:1, IV:2, and IV:3, according to the method previously described by Torres et al. [35]. Interval used as reference was 70-126 nmol/h/mg hemoglobin.

\section{PRPS1 expression analysis}

PRPS1 expression analysis was performed in RNA samples using blood lymphocytes from III:2, IV:1, IV:2, and 
IV:3. Total RNA was reversely transcribed to cDNA with ImProm-II ${ }^{\mathrm{mm}}$ Reverse Transcription System (Promega) using random primers. RT-PCR experiments were performed using PRPS1 exonic primers pairs spanning exons $1-2,1-4$, and 4-7 (primers available in the Additional file 1). Primers for the housekeeping GAPDH gene were used as internal control. PCR fragments were subjected to electrophoresis in a gel searching for abnormally spliced amplicons and further sequenced.

\section{X-chromosome inactivation}

$\mathrm{X}$ chromosome inactivation assay was performed in sodium bisulfite-treated genomic DNA from peripheral blood and saliva (EpiTect Bisulfite Kit, Quiagen) by a methylation specific PCR of the first exon of the human androgen receptor locus with fluorochrome-coupled primers. Heterozygosity of the human androgen receptor region in the target samples was previously checked. Two different pairs of primers were used to detect the methylated and unmethylated alleles and PCR fragments were analyzed in an ABI PRISM 3100 Genetic Analyzer (Applied Biosystems). X chromosome inactivation skewing was reported as percentage of the predominant allele and was considered skewed when the predominant allele exceeded $74 \%$, non-skewed between $50 \%$ and $65 \%$ and undetermined between $66 \%$ and $74 \%$ [36].

\section{Results}

\section{Familial history of RP-0482}

Data from ophthalmological, neurological and otological examinations of patients III:2, IV:2, and IV:3 were available from a period of more than 15 years and the phenotype is described in detail in the Additional file 1. Patient II:2 also displayed sectorial RP and ataxic traits, but the progression of the clinical phenotype is not available. Figure 2 shows the retinal and MRI images of the three affected females. Age of onset, presentation and severity of the phenotype are highly variable in the family, as summarized in Table 1 . Both the index patient and her mother have various degrees of ataxia, peripheral neuropathy and hearing loss beyond the ophthalmological symptoms, whereas the phenotype of the affected sister is currently confined to the eye and milder than those of III:2 and IV:3 (Additional files 1 and 2)

Identification of a novel missense mutation in PRSP1 by WES DNA samples from individuals III:3, IV:2, and IV:3 were subjected to WES (Figure 1A). A total of 10.77 GB data on target genomic regions was generated for the three samples, with a mean coverage of target region of 78.23X. An average of 48,306 SNVs and 8,218 insertion/deletion (indels) were called for the three exomes, but for further filtering only coding variants were considered, thus reducing the number to an average of 18,722 SNVs and 741 indels per sample. Variant filtering under the assumption of a dominant inheritance yielded 141 variants in 126 genes as potential candidates (Additional file 3) with no known cause of retinal degeneration amongst them (RetNet, [28] accessed June, 2013). Further filtering of the 141 variants, left 12 novel variants, conserved across species according to the values from PhyloP and GERP++, predicted to be pathogenic at least by two of the systems evaluated and with expression in the retina, so they were selected for validation and segregation in the family (Additional file 1). Only the novel missense change in exon 1 of PRPS1, c.46 T > C; p.Ser16Pro (NM_001204402), completely segregated in all four affecteds and eight unaffected members of family RP-0482 (Figure 1A) and was absent in controls (258 X chromosomes total). Both GERP++ and PhyloP values estimated a high degree of conservation of serine at position 16 of PRS-I across species (Additional file 3), and was confirmed by multiple sequence alignment (Figure 1B), being the first missense mutation identified in this gene from the first $\alpha$-helix to the fourth $\beta$-strand (aminoacids 1 to 43, Figure 1C). All in silico programs evaluated except for Polyphen 2 predicted $\mathrm{p}$. Ser16Pro to be damaging (Additional file 3). The tridimesional model of PRS-I with p.Ser16Pro indicated that Ser16 is located in the first $\alpha$-helix of the protein in the $\mathrm{N}$-terminal domain (Figure 1D). The replacement of serine with proline leads to the loss of a hydrogen bond with Gln13 likely breaking the tightly packed $\alpha$-helix.

\section{p.Ser16Pro leads to PRS deficiency in females}

To further assess the functional effect of the mutation in vitro, PRS enzymatic activity was determined in erythrocytes from three affected females (III:2, IV:2, and IV:3), where different levels of enzyme deficiency were evidenced (Table 1), and two unaffecteds (III:3 and IV:1) with PRS-I within the normal range.

\section{Lack of expression of PRPS1 p.Ser16 in the index patient}

The in silico analysis using ESEFinder predicted this mutation to alter the recognition pattern of splicing RNA proteins compared to the wild type sequence. To confirm this, PRPS1 expression analysis was performed in RNA samples from blood lymphocytes from three affecteds and one unaffected (IV:1). RT-PCR analysis yielded no differences on the expression of PRPS1 transcripts between carriers of p.Ser16Pro and the non-carrier (Figure 1E), and no additional splicing transcripts were found. Notably, further sequencing of mRNA transcripts evidenced the mutation in homozygosis in the index patient (Figure 1E). 


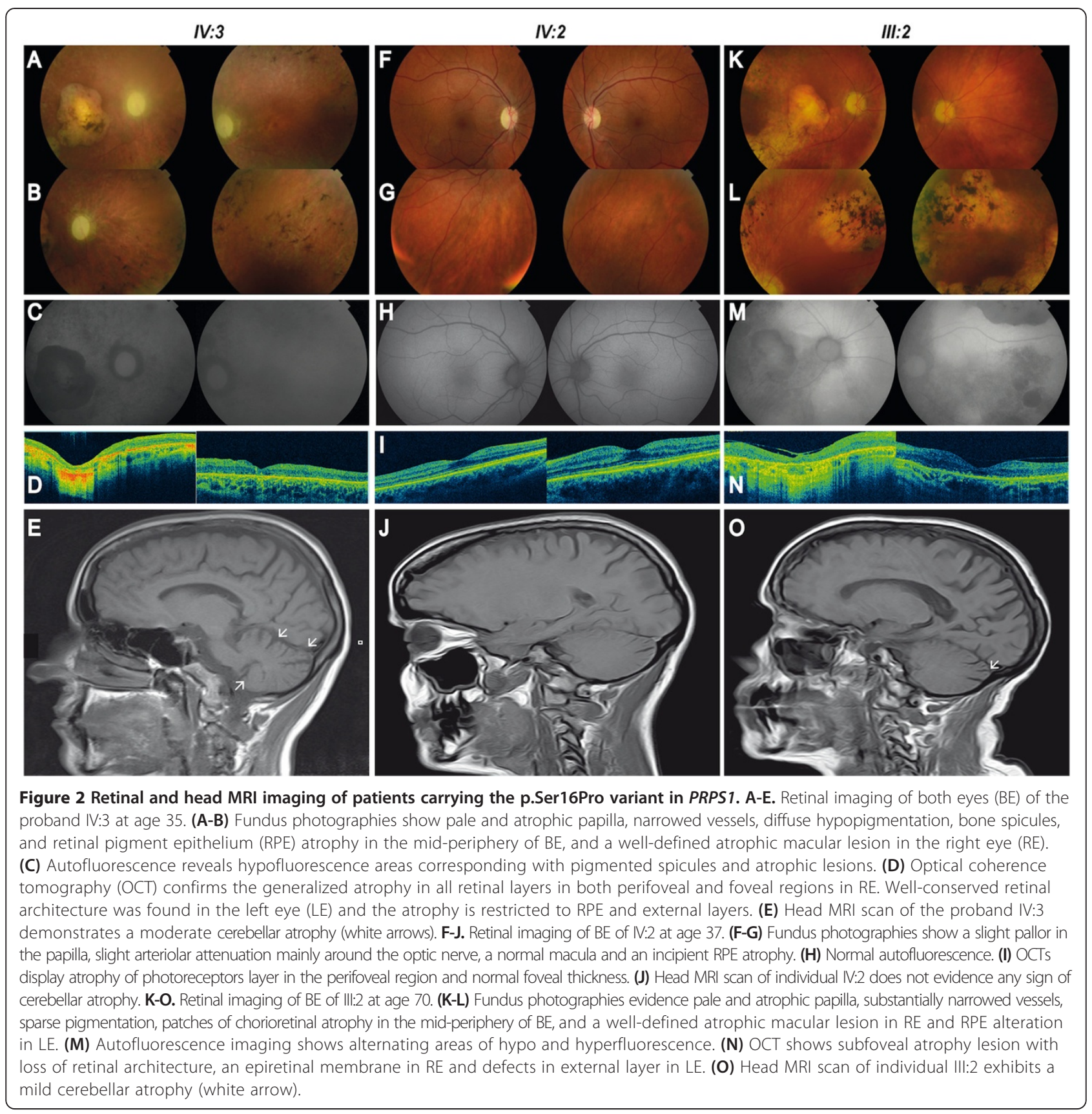

\section{Non-random patterns of $\mathrm{X}$-chromosome inactivation in the index patient}

Non-random patterns of X-chromosome inactivation were assessed in the three affecteds (III:2, IV:2 and IV:3) and one unaffected individual (IV:1). Only in IV:3, there was a significantly skewed inactivation $(82 \%)$ of the paternal allele, which explains the lack of expression of the wild type allele observed in the mRNA (Figure 1E). Individuals IV:1, IV:2 and III:2, had 50\%, 61\% and $64 \% \mathrm{X}$-chromosome inactivation, respectively, so they were considered non-skewed.

\section{Discussion}

Here we report a novel mutation in PRPS1 leading to PRS-I deficiency in three-females from a family with a phenotype consisting of OA followed by RP in all cases, plus neurological features overlapping CMTX5 and Arts syndrome with variable presentation in the proband (IV:3) and her mother (III:2).

Despite being already described that carrier females of PRPS1 mutations can exhibit PRS-I deficiency and a disease phenotype [3,7-9], to date, no female has been reported to display such a complex and severe phenotype 
Table 1 Summary of findings in family RP-0482 and comparison with other PRS-I deficiency disorders (adapted from de Brouwer et al. [11])

\begin{tabular}{|c|c|c|c|c|c|c|}
\hline & IV:3 & IV:2 & III:2 & Arts syndrome & CMTX5 & DFN2 \\
\hline Date of birth & 1978 & 1976 & 1943 & & & \\
\hline \multicolumn{7}{|l|}{ Symptoms (age at diagnosis) } \\
\hline \multicolumn{7}{|l|}{ Ophthalmological } \\
\hline Retinitis Pigmentosa & $+(14 y)$ & $+(23 y)$ & $+(47 y)$ & - & - & - \\
\hline Night blindness & $++(4 y)$ & $+(16 y)$ & + & - & - & - \\
\hline Visual field constriction & ++ & + & + & - & - & - \\
\hline Visual acuity loss & $++(4 y)$ & - & ++ & - & - & - \\
\hline ERG alteration & ++ & $+/-$ & ++ & - & - & - \\
\hline Pigmentary changes at fundus & + & $+/-$ & + & - & - & - \\
\hline Macular atrophy & ++ & + & + & - & - & - \\
\hline Optic atrophy & $+(5 y)$ & $+(16 y)$ & $+(47 y)$ & + & $+(\sim 10 \mathrm{y})$ & + \\
\hline Nystagmus & $+($ Congenital) & - & - & - & - & - \\
\hline Cataracts & + & - & + & - & - & - \\
\hline Hyperopia & + & + & $+/-$ & - & - & - \\
\hline Hearing impairment & $++(21 y)$ & - & $+(50 y)$ & ++ & + & + \\
\hline Neurological & $++(34 \mathrm{y})$ & - & $+(55 y)$ & & & \\
\hline Mild developement delay & $+/-$ & - & - & + & - & - \\
\hline Hypotonia & + & - & - & + & + & - \\
\hline Delayed motor development & - & - & - & + & - & - \\
\hline Peripheral neuropathy & ++ & - & + & + & + & - \\
\hline Pes cavus & + & - & - & NR & NR & NR \\
\hline Loss of deep tendom reflexes & - & - & - & - & + & - \\
\hline Cerebellar Atrophy & ++ & - & + & & & \\
\hline Ataxia & + & - & + & + & + & - \\
\hline Essential tremor & + & + & + & & & \\
\hline Symptoms in carrier females & +++ & + & ++ & Isolated and milder & Hearing loss & None \\
\hline \multirow{3}{*}{$\begin{array}{l}\text { PRS activity erythrocytes } \\
\text { (nmol/h/mg Hb) } \\
\text { (Reference: 70-126) }\end{array}$} & \multirow[t]{3}{*}{ Erythrocytes: 10} & \multirow[t]{3}{*}{ Erythrocytes: 41} & \multirow[t]{3}{*}{ Erythrocytes: 65} & $\begin{array}{l}\text { Erythrocytes: } \\
\text { No activity }\end{array}$ & \multirow[t]{3}{*}{$\begin{array}{l}\text { Fibroblasts: } \\
\text { Decreased }\end{array}$} & \multirow{3}{*}{$\begin{array}{l}\text { Erythrocytes anc } \\
\text { fibroblasts: } \\
\text { Decreased }\end{array}$} \\
\hline & & & & $\begin{array}{l}\text { Fibroblasts 13-fold } \\
\text { decrease }\end{array}$ & & \\
\hline & & & & EBV-LCLs: normal & & \\
\hline Structural effect of mutation & \multicolumn{3}{|c|}{ Whole protein structure? } & $\begin{array}{l}\text { ATP site and allosteric } \\
\text { sites I and || }\end{array}$ & $\begin{array}{l}\text { ATP site and } \\
\text { allosteric site I }\end{array}$ & Local structure \\
\hline
\end{tabular}

The number of " + " indicate the severity of the manifestation. NR = Not reported and EBV-LCLs = Epstein-Barr virus-transformed lymphoblastoid cell lines.

as observed in this family, specially in the proband. This patient showed a phenotype with features of Arts syndrome and CMTX5, with optic atrophy as the first symptom, followed by RP and neurological manifestations, such as hearing loss, intellectual disability and peripheral neuropathy (Table 1). The findings from this patient are consistent with the increasing evidence of intermediate phenotypes in PRS-I syndromes, recently described by several authors $[6,7,12,37]$. In particular, Synofzik and colleagues recently described a male with an intermediate phenotype between CMTX5 and Arts syndrome and a carrier female affected with DFN2 due to X-chromosome inactivation skewing [7]. The authors observed a correlation between the enzymatic residual activity, the degree of $\mathrm{X}$ chromosome inactivation skewing and the phenotype in the female and postulated that the location of the mutation and the residual enzymatic activity would be the main determinants of the phenotypic manifestations in males and females, respectively [7]. The three affected females from our family exhibited different levels of PRS deficiency in erythrocytes, ranging from a severe decrease in the activity in IV:3 to an 
almost normal activity in III:2. However, non-random patterns of X-chromosome inactivation were exclusively observed for the index patient (IV:3), who was found to only express the mutant allele in lymphocytes. In this patient, there was an apparent correlation between the $\mathrm{X}$-chromosome inactivation in leukocytes, the lack of expression of the wild type allele in lymphocytes, the degree of PRS deficiency in erythrocytes, and the severity of the phenotype. $\mathrm{X}$ chromosome inactivation in subjects III:2 and IV:2 was not skewed and the degree of PRS-I deficiency only correlated with the age of onset of ophthalmological symptoms but not with the presentation or severity of the phenotype. This lack of correlation is not surprising given that $\mathrm{X}$-inactivation, whether random or biased, occurs in a tissue-specific manner and what is observed in a particular tissue or cell type, such as leukocytes may not be representative of the status of central and peripheral nervous systems $[7,38]$, which are primarily affected in PRS-I deficiency syndromes. Given the variable expression of the disease in the family it is very possible that either $\mathrm{X}$-inactivation is skewed in those target systems or that the expression of PRS-II is compensating PRS enzymatic function as previously suggested, and so phosphoribosyl pyrophosphate synthesis would not be critically affected [11].

A relationship between the level of PRS-I disruption and the different syndromic manifestations has been suggested [11], with the most severe syndromes caused by mutations predicted to impact allosteric and active sites and those responsible for milder phenotypes disrupting the structure locally [11]. Very recently, AlMaawali and colleagues expanded the PRPS1 phenotype to retinal dystrophy and diabetes insipidus in a family with two males affected with Leber's congenital amaurosis along with other manifestations, and no carrier female affected [37]. The mutation responsible for the phenotype was located in exon 5 of the gene (p.Arg196Trp), close to the ATP binding site of PRS-I. Residue Ser16 is located in the $\mathrm{N}$-terminal domain of the protein, in a region not previously found mutated (Figure 1C) and far from the allosteric and active sites, both associated with Arts syndrome, CMTX5, and the recent retinal dystrophy phenotype, all features harbored by this family. That demonstrates once again the mutation-specific nature of PRS-I phenotypes $[7,11]$. Nevertheless, the existence of a putative defective splicing isoform, as predicted by in silico analysis cannot be ruled out, as it would be degraded by nonsense-mediated decay and thus, would not be detected by RT-PCR. In addition, other genes or epigenomic factors could be contributing to the complexity and severity of the phenotypes of PRS-I syndromes. Finally, it is worth noting that we cannot rule out either a dominant inheritance pattern for this particular mutation with a lethal effect in hemizygotes due to the lack of affected males in the family.

\section{Conclusion}

These results support previous findings evidencing the existence of intermediate phenotypes in PRS-I deficiency syndromes and demonstrate that female carriers of PRPS1 mutations can be as severely affected as their male counterparts and therefore, these syndromes may need also to be considered in females even in the absence of affected males in the family.

\section{Additional files}

\begin{abstract}
Additional file 1: Clinical data of family RP-0482 and primers used in the study.

Additional file 2: Figure S1. Multifocal ERG and Optical coherence tomography $(\mathrm{OCT})$ findings suggested a sectorial affectation of perifoveal photoreceptors. A. Right eye multifocal ERG (mfERG) records in patient $\mathrm{N}$ :2 demonstrate a reduced function of photoreceptors of perifoveal region with preserved function of fovea. The ring analysis (see schematic) goes from the centre to the periphery. Quantitative results of mfERG analyses are displayed in table format. B. OCT macular cube $512 \times 128$ scan show macular thickness in the right eye from patient IV:2. ILM: inner limiting membrane; RPE: retinal pigment epithelium. Top left: fundus image with scan cube overlay. Top right: macular thickness significance map. The central innermost 1-mm-diameter circle represents the central subfield; inner superior, inner nasal, inner inferior, and inner temporal areas bounded by the 3-mm-diameter circle form the inner macula; outer superior, outer nasal, outer inferior, and outer temporal areas bounded by the 6-mm-diameter circle form the outer macula. Retinal thickness values from ILM to RPE are compared to the normative data. Middle and bottom left: cross-sectional OCT scans. Middle right: 3 days surface maps: the ILM-RPE, displaying the retinal thickness in three dimensions. Bottom right: central subfield thickness, overall average macular thickness, and overall macular volume compared to normative data are displayed in table format. Reduction in the retinal thickness in the perifoveal region with a normal foveal thickness is also evidenced.
\end{abstract}

Additional file 3: Table S1 and S2. Genetic variants obtained (single nucleotide variants -SNV-, Table S1, and insertion deletion variants -indels-, Table S2) after the first variant filtering analysis. Table S3. Variants yielded by the second filtering of the 141 variants based on the novelty, conservation across species according to the values from PhyloP and GERP++, predicted pathogenicity at least by two of the systems evaluated and expression in the retina. 1KGP: Minor allele frequency in the 1000 Genome Project, ESP: Minor allele frequency in the NHLBI GO Exome Sequencing Project. For PhyloP prediction, C: Conserved, NC: Not conserved. For pathogenicity prediction systems, B: benign, D: deleterious (LRT), or damaging (SIFT) or disease causing (MutationTaster), N: neutral (LRT) or polymorphism (MutationTaster), NA: missing data, PrD: probably damaging, PsD: possibly damaging, T: tolerated, U: Unknown.

\section{Abbreviations}

CMTX5: X-linked Charcot-Marie-Tooth, type 5; DFN2: X-linked non-syndromic sensorineural deafness; Indel: Insertion/deletion polymorphism; OA: Optic atrophy; PRPP: Phosphoribosyl pyrophosphate; PRS: Phosphoribosyl pyrophosphate synthetase; RP: Retitinis pigmentosa; SNV: Nonsynonymous single nucleotide variant; WES: Whole exome sequencing.

\section{Competing interests}

The authors declare that they have no competing interests.

\section{Authors' contributions}

BA contributed to the design of the study, performed experiments and data analysis, the interpretation of results, and wrote the manuscript. SH contributed to the design of the study, performed the whole exome sequencing and helped with the data analysis. MC and PFS contributed to the design of the study, performed experiments and data analysis, interpretation of the results and wrote the manuscript. FBK, MILM, BGS, JdV 
performed the clinical evaluation of the patients and the interpretation of the phenotypic analysis. YG and LT contributed to the design of the study, performed data analysis and interpretation of the results. $X \mathrm{~L}, \mathrm{LG}$, and $X X$ performed the whole exome sequencing and helped with the data analysis. RJT and JGP performed experiments, data analysis and interpretation of results. $\mathrm{HH}$ contributed to the design of the study, interpretation of the results and writing of the manuscript. BK contributed to the design of the study and helped in the data analysis. CA contributed to the design of the study, the clinical evaluation of the patients; the interpretation of the results and the writing of the manuscript. All authors contributed to the writing and critical review of this manuscript. All authors read and approved the final manuscript.

\section{Acknowledgements}

We would like to thank all individuals participating in this study. We also want to thank FJD-Biobank (RD09/0076/00101), CIBER-ER (06/07/0036), FIS (PI:13/00226), ONCE 2013 and Fundaluce (4019-002) for their support. Patricia Fernandez San Jose's work is supported by a Rio Hortega grant (CM12/00013) and Marta Corton by a Miguel Servet grant (CP/03256), both from Instituto de Salud Carlos III. This work was funded by the Kubert Estate Gift fund, the National Human Genome Research Institute (\# U01 HG006830), and also by Institutional Development Funds to the Center for Applied Genomics (CAG) at the Children's Hospital of Philadelphia (CHOP). This study was supported by the Shenzhen Municipal Government of China (No. GJHZ20130417140916986)

\section{Author details}

${ }^{1}$ Center for Applied Genomics, The Children's Hospital of Philadelphia, Philadelphia, PA 19104, USA. ${ }^{2}$ College of Life Sciences, University of Chinese Academy of Sciences, Beijing 100049, China. ${ }^{3}$ BGI-Shenzhen, Shenzhen 518083, China. ${ }^{4}$ Department of Genetics and Genomics, IIS-Fundación Jiménez Díaz University Hospital (IISFJD, UAM), 28040 Madrid, Spain. ${ }^{5}$ Center for Biomedical Network Research on Rare Diseases (CIBERER), ISCIII, Madrid, Spain. ${ }^{6}$ Department of Ophthalmology, Fundación Jiménez Díaz, 28040 Madrid, Spain. ${ }^{7}$ Department of Neurology, Fundación Jiménez Díaz, 28040 Madrid, Spain. ${ }^{8}$ Department of Biochemistry, La Paz University Hospital IdiPaz, Madrid 28046, Spain. ${ }^{9}$ Department of Internal Medicine, Metabolic-Vascular Unit, La Paz University Hospital IdiPaz, Madrid 28046, Spain. ${ }^{10}$ The Guangdong Enterprise Key Laboratory of Human Disease Genomics, Shenzhen, China.

Received: 26 June 2014 Accepted: 11 November 2014 Published online: 10 December 2014

\section{References}

1. Roessler BJ, Bell G, Heidler S, Seino S, Becker M, Palella TD: Cloning of two distinct copies of human phosphoribosylpyrophosphate synthetase cDNA. Nucleic Acids Res 1990, 18(1):193.

2. Taira M, Ishijima S, Kita K, Yamada K, lizasa T, Tatibana M: Nucleotide and deduced amino acid sequences of two distinct cDNAs for rat phosphoribosylpyrophosphate synthetase. J Biol Chem 1987, 262(31):14867-14870.

3. de Brouwer AP, Williams KL, Duley JA, van Kuilenburg AB, Nabuurs SB, Egmont-Petersen M, Lugtenberg D, Zoetekouw L, Banning MJ, Roeffen M, Hamel BC, Weaving L, Ouvrier RA, Donald JA, Wevers RA, Christodoulou J, van Bokhoven $\mathrm{H}$ : Arts syndrome is caused by loss-of-function mutations in PRPS1. Am J Hum Genet 2007, 81(3):507-518.

4. Kim HJ, Sohn KM, Shy ME, Krajewski KM, Hwang M, Park JH, Jang SY, Won HH, Choi BO, Hong SH, Kim BJ, Suh YL, Ki CS, Lee SY, Kim SH, Kim JW: Mutations in PRPS1, which encodes the phosphoribosyl pyrophosphate synthetase enzyme critical for nucleotide biosynthesis, cause hereditary peripheral neuropathy with hearing loss and optic neuropathy (cmtx5). Am J Hum Genet 2007, 81(3):552-558.

5. Liu X, Han D, Li J, Han B, Ouyang X, Cheng J, Li X, Jin Z, Wang Y, Bitner-Glindzicz M, Kong X, Xu H, Kantardzhieva A, Eavey RD, Seidman CE, Seidman JG, Du LL, Chen ZY, Dai P, Teng M, Yan D, Yuan H: Loss-of-function mutations in the PRPS1 gene cause a type of nonsyndromic X-linked sensorineural deafness, DFN2. Am J Hum Genet 2010, 86(1):65-71.

6. Park J, Hyun YS, Kim YJ, Nam SH, Kim SH, Hong YB, Park JM, Chung KW, Choi BO: Exome Sequencing Reveals a Novel Mutation in a Family with CMTX5 without Optic Atrophy. J Clin Neurol 2013, 9(4):283-288.
7. Synofzik M, Muller vom Hagen J, Haack TB, Wilhelm C, Lindig T, Beck-Wodl S, Nabuurs SB, van Kuilenburg AB, de Brouwer AP, Schols L: X-linked Charcot-Marie-Tooth disease, Arts syndrome, and prelingual non-syndromic deafness form a disease continuum: evidence from a family with a novel PRPS1 mutation. Orphanet journal of rare diseases 2014, 9:24.

8. Rosenberg RN, Chutorian A: Familial opticoacoustic nerve degeneration and polyneuropathy. Neurology 1967, 17(9):827-832.

9. Pauli RM: Sensorineural deafness and peripheral neuropathy. Clin Genet 1984, 26(4):383-384.

10. Arts WF, Loonen MC, Sengers RC, Slooff JL: X-linked ataxia, weakness, deafness, and loss of vision in early childhood with a fatal course. Ann Neurol 1993, 33(5):535-539.

11. de Brouwer AP, van Bokhoven H, Nabuurs SB, Arts WF, Christodoulou J, Duley J: PRPS1 mutations: four distinct syndromes and potential treatment. Am J Hum Genet 2010, 86(4):506-518.

12. Moran R, Kuilenburg AB, Duley J, Nabuurs SB, Retno-Fitri A, Christodoulou J, Roelofsen J, Yntema HG, Friedman NR, van Bokhoven H, de Brouwer AP: Phosphoribosylpyrophosphate synthetase superactivity and recurrent infections is caused by a p.Val142Leu mutation in PRS-I. Am J Med Genet A 2012, 158A(2):455-460.

13. Garcia-Pavia P, Torres RJ, Rivero M, Ahmed M, Garcia-Puig J, Becker MA: Phosphoribosylpyrophosphate synthetase overactivity as a cause of uric acid overproduction in a young woman. Arthritis Rheum 2003, 48(7):2036-2041.

14. ClustalW2, Multiple Sequence Alignment: http://www.ebi.ac.uk/Tools/msa/ clustalw2/.

15. Li H, Durbin R: Fast and accurate short read alignment with Burrows-Wheeler transform. Bioinformatics 2009, 25(14):1754-1760.

16. McKenna A, Hanna M, Banks E, Sivachenko A, Cibulskis K, Kernytsky A, Garimella K, Altshuler D, Gabriel S, Daly M, DePristo MA: The Genome Analysis Toolkit: a MapReduce framework for analyzing next-generation DNA sequencing data. Genome Res 2010, 20(9):1297-1303.

17. Wang K, Li M, Hakonarson H: ANNOVAR: functional annotation of genetic variants from high-throughput sequencing data. Nucleic Acids Res 2010, 38(16):e164.

18. Kumar P, Henikoff S, Ng PC: Predicting the effects of coding non-synonymous variants on protein function using the SIFT algorithm. Nat Protoc 2009, 4(7):1073-1081

19. Adzhubei IA, Schmidt S, Peshkin L, Ramensky VE, Gerasimova A, Bork P, Kondrashov AS, Sunyaev SR: A method and server for predicting damaging missense mutations. Nat Methods 2010, 7(4):248-249.

20. Chun S, Fay JC: Identification of deleterious mutations within three human genomes. Genome Res 2009, 19(9):1553-1561.

21. Schwarz JM, Rodelsperger C, Schuelke M, Seelow D: MutationTaster evaluates disease-causing potential of sequence alterations. Nat Methods 2010, 7(8):575-576.

22. Siepel A, Pollard K, Haussler D: New Methods for Detecting LineageSpecific Selection. In RECOMB'06 Proceedings of the 10th International Conference on Research in Computational Molecular Biology. Heidelberg: Springer-Verlag Berlin; 2006:190-205.

23. Davydov EV, Goode DL, Sirota M, Cooper GM, Sidow A, Batzoglou S: Identifying a high fraction of the human genome to be under selective constraint using GERP++. PLoS Comput Biol 2010, 6(12):e1001025.

24. Liu X, Jian X, Boerwinkle E: dbNSFP: a lightweight database of human nonsynonymous SNPs and their functional predictions. Hum Mutat 2011, 32(8):894-899.

25. 1000 Genomes Project: http://www.1000genomes.org/

26. NHLBI GO Exome Sequencing Project: http://evs.gs.washington.edu/EVS/

27. The Human Gene Mutation Database: http://www.hgmd.org/

28. The Retinal Information Network (RetNet): https://sph.uth.edu/RetNet/ home.htm.

29. Online Mendelian Inheritance in Man: http://www.omim.org/.

30. Arnold K, Bordoli L, Kopp J, Schwede T: The SWISS-MODEL workspace: a web-based environment for protein structure homology modelling. Bioinformatics 2006, 22(2):195-201.

31. Swiss Model: http://swissmodel.expasy.org/

32. Swiss PDB viewer: http://spdbv.vital-it.ch/

33. ESEFinder: http://rulai.cshl.edu/cgi-bin/tools/ESE3/esefinder.cgi? process=home

34. Cartegni L, Wang J, Zhu Z, Zhang MQ, Krainer AR: ESEfinder: A web resource to identify exonic splicing enhancers. Nucleic Acids Res 2003, 31(13):3568-3571. 
35. Torres RJ, Mateos FA, Puig JG, Becker MA: A simplified method for the determination of phosphoribosylpyrophosphate synthetase activity in hemolysates. Clin Chim Acta 1994, 224(1):55-63.

36. Bolduc V, Chagnon P, Provost S, Dube MP, Belisle C, Gingras M, Mollica L, Busque $L$ : No evidence that skewing of $X$ chromosome inactivation patterns is transmitted to offspring in humans. J Clin Invest 2008, 118(1):333-341.

37. Al-Maawali A, Dupuis L, Blaser S, Heon E, Tarnopolsky M, Al-Murshedi F, Marshall CR, Paton T, Scherer SW, for the FCC, Roelofsen J, van Kuilenburg AB, Mendoza-Londono R.: Prenatal growth restriction, retinal dystrophy, diabetes insipidus and white matter disease: expanding the spectrum of PRPS1-related disorders. Eur J Hum Genet 2014. [Epud ahead of print]

38. Van den Veyver IB: Skewed X inactivation in X-linked disorders. Semin Reprod Med 2001, 19(2):183-191.

doi:10.1186/s13023-014-0190-9

Cite this article as: Almoguera et al:: Expanding the phenotype of PRPS1 syndromes in females: neuropathy, hearing loss and retinopathy. Orphanet Journal of Rare Diseases 2014 9:190.

\section{Submit your next manuscript to BioMed Central and take full advantage of:}

- Convenient online submission

- Thorough peer review

- No space constraints or color figure charges

- Immediate publication on acceptance

- Inclusion in PubMed, CAS, Scopus and Google Scholar

- Research which is freely available for redistribution 